\title{
¿Qué se puede aprender «Jugando con la electricidad» en Educación Infantil?
}

\author{
Javier Rodríguez Moreno \\ Departamento de Pedagogía. Universidad de Jaén.España.jrmoreno@ujaen.es \\ ORCID: https:/ / orcid.org/0000-0002-5890-3654
}

Carlos de Pro Chereguini

Departamento de Didáctica de las Ciencias Experimentales. Universidad de Murcia. España.cpro@um.es ORCID: https:/ / orcid.org/0000-0002-4148-2910

\author{
Antonio de Pro Bueno \\ Departamento de Didáctica de las Ciencias Experimentales. Universidad de Murcia. España.nono@um.es \\ ORCID: https:/ / orcid.org/0000-0003-3301-8787
}

[Recibido: 1 Octubre 2019. Revisado: 11 Diciembre 2019. Aceptado: 2 Enero 2020]

\begin{abstract}
Resumen: El trabajo presenta una experiencia llevada a cabo en Segundo Ciclo de Educación Infantil en un centro público de Andalucía. Partiendo de la necesidad de diseñar, aplicar y evaluar propuestas para esta etapa educativa, nos hemos planteado: ¿Cómo se desarrolló y qué efectos produjo, desde el punto de vista del aprendizaje del alumnado, la propuesta «Jugando con la electricidad» en un aula de Educación Infantil? Se fundamenta y presenta la unidad didáctica, los resultados de su puesta en práctica en dos aulas y las conclusiones que hemos extraído de nuestra experiencia.
\end{abstract}

Palabras clave: Educación Infantil; Propuesta de enseñanza; Circuitos eléctricos; Medidas de sostenibilidad y seguridad; Aprendizaje del alumnado.

\section{What can we learn «Playing with electricity» in Early Childhood Education?}

\begin{abstract}
The work presents an experience carried out in the Second Cycle of Early Childhood Education in a public centre in Andalusia. Starting from the need to design, apply and evaluate proposals for this educational stage, we have wondered: How was produced the proposal «Playing with electricity» in a classroom from the point of view of student learning of Early Childhood Education? We base and present the teaching unit, the results of its implementation in two classrooms and the conclusions we have drawn from our experience.
\end{abstract}

Keywords: Early Childhood Education; Teaching Proposal; Electrical circuits; Sustainability and security measures; Student learning.

Para citar este artículo: Rodríguez-Moreno J., Pro-Chereguini C., Pro-Bueno A. (2020) ¿Qué se puede aprender «Jugando con la electricidad» en Educación Infantil?. Revista Eureka sobre Enseñanza y Divulgación de las Ciencias 17(2), 2202. doi: 10.25267/Rev_Eureka_ensen_divulg_cienc.2020.v17.i2.2203

\section{Origen y justificación del trabajo}

Una de las características más destacables de nuestro sistema educativo es su permanente estado de cambio curricular: reformas, contrarreformas, reformas de contrarreformas... Sin embargo, si hay una etapa educativa en la que existe una cierta estabilidad, ésta es la de Educación Infantil (en adelante EI). ¿Es fruto del acierto de los legisladores? ¿Es el resultado de que las maestras de este nivel educativo saben qué hacer con independencia de los programas oficiales? ¿Es que no ha habido una evolución en la investigación e innovación en este ámbito?... Lo cierto es que, con independencia de la estabilidad de las reformas, creemos que los auténticos gestores de las mismas somos los docentes, como ya decía Fullan (1994, 2008) y nosotros somos los que las traducimos en las aulas y los centros.

Revista Eureka sobre Enseñanza y Divulgación de las Ciencias

Universidad de Cádir: APAC-Eureka. ISSN: 1697-011X 
En los libros de texto -uno de los traductores más utilizados de cualquier reformaencontramos ilustraciones modernas y atractivas, con más calidad; formatos que han podido mejorar; la incorporación de material de apoyo en las páginas de las editoriales... pero los contenidos siguen siendo los de siempre, estáticos, difíciles de justificar para el alumnado de estas edades (y probablemente no sólo de éstas), enlatados en actividades poco motivadoras... Parece que muchos de estos materiales han sido impermeables a las transformaciones experimentadas en la sociedad: los nuevos retos de la ciudadanía, las aportaciones de las tecnologías de la información y la comunicación, los cambios en la forma de aprender, la creciente interculturalidad... Parece que se dirigieran a niños y niñas de otra época.

No obstante, tanto antes como ahora, siempre ha habido un cierto consenso en la finalidad de esta etapa educativa: la importancia de desarrollar las potencialidades del alumnado. Un alumnado que es obligadamente heterogéneo, porque en sí mismos tienen características personales distintas, porque han crecido en contextos familiares muy diferentes, porque han tenido distintas experiencias de vida a pesar del escaso tiempo trascurrido desde que nacieron... De esta manera, la etapa de EI deja de ser un periodo de formación para el futuro y pasa a ser uno para el presente.

Los estudiantes necesitan encontrar una utilidad a lo que deben aprender en el aula que vaya más allá de la escuela y, por supuesto, conectar lo que estudian con lo que viven: con los dibujos animados que ven en la televisión, con lo que leen o les leen en los cómics o en los cuentos, con sus inquietudes y preocupaciones infantiles, con el estilo de vida que están desarrollando en sus casas, etc. Pero, además, necesitamos fomentar la capacidad de asombrarse, la creatividad, el disfrutar en la escuela, la presencia de las emociones, la experimentación como antesala del descubrimiento, la necesidad de cooperar... Pensamos que estos aspectos no pueden olvidarse en las clases de EI, porque posiblemente sean algunas razones que justifiquen su existencia.

Para ello, hemos de plantear situaciones de aprendizaje, experiencias o vivencias, en las que no sólo se aprendan cosas, sino que se aprendan a hacer cosas, a hacer cosas con otros, a saber cuándo y para qué hacerlas... En este sentido, creemos que el Área de Conocimiento del Entorno ofrece unas posibilidades únicas, derivadas de la naturaleza de sus propios contenidos. Eso sí, necesitamos actividades en las que el alumnado no se aburra, se encuentre interesado, participe, aprenda de forma divertida... En definitiva, dejar de hacer fichas, desmotivadoras y descontextualizadas, y rescatar la idea del desarrollo socio-cognitivo (Hadzigeorgiou 2002, Eshach y Fried 2005, Cobe 2015) o su versión evolucionada, el culturalcognitivo (Fragkiadaki y Ravanis 2014, Fleer 2015).

En este contexto, hemos diseñado una unidad didáctica, la hemos llevado a unas aulas de EI y nos hemos planteado: ¿Cómo se desarrolló y qué efectos produjo, desde el punto de vista del aprendizaje del alumnado, la propuesta «Jugando con la electricidad»?

\section{Revisión de trabajos realizados en este ámbito}

Las investigaciones realizadas en la Didáctica de las Ciencias Experimentales en España se han preocupado mucho más de otras etapas educativas que de la EI, lo que refuerza la necesidad de trabajos como el nuestro (García 2008, Pro y Rodríguez 2011, Benarroch 2012).

En cuanto a propuestas de enseñanza, aunque cualquier trabajo siempre nos aporta ideas, hay variables que complican la transferencia inter-contextual: las diferencias entre currículos, en la formación del profesorado, en la cultura escolar... y, sobre todo, en las características del alumnado. No obstante, nos han parecido esclarecedores trabajos como los de Kamii y Devries (1983), Moreno (1986), Tonucci (1997), Kaufman y Fumagalli (2000), French (2004), 
Vega (2006, 2008), Hecker (2008), Laguía y Vidal (2008), Vizcaíno (2008), Sugrañez et al. (2012), Cantó, Pro y Solbes (2016)... A estos habría que añadir proyectos como La main à la pate ${ }^{2}$ el Programa Perseo ${ }^{2}$ o el Proyecto Pollen ${ }^{3}$. Así mismo, hay aportaciones de instituciones de educación no formal como el Museu de la Ciencia de la Caixa (2002) o del Pequemuseo del Museo Nacional de Ciencia y Tecnología (2007), que también pueden ser una fuente para idear propuestas.

A la hora de elegir una temática, queríamos optar por alguna con una presencia en la vida cotidiana del niño de estas edades, que aportara conocimientos para cubrir necesidades básicas de un ciudadano (sostenibilidad, seguridad, consumo saludable...), que contemplara actividades experimentales, que exigiera una participación activa del estudiante, que integrara fácilmente tareas de cooperación con los compañeros, que posibilitara la participación de las madres y padres... y que tuviera un cierto grado de innovación en esta etapa educativa. Aunque hay muchos tópicos que reúnen estas características, elegimos los circuitos eléctricos.

Nuestra intención ha sido que la propuesta a ensayar fuera coherente con los avances de la Didáctica de las Ciencias Experimentales y utilizara algunos planteamientos innovadores de la misma. No obstante, no hemos obviado el currículo oficial (BOJA 2008); en éste, se plantean tres áreas, para alcanzar unas capacidades determinadas al acabar la etapa.

En todo caso, como cualquier otro currículo oficial, no nos informa los contenidos concretos que había que enseñar y mucho menos de cómo hacerlo o de cómo valorar el aprendizaje del alumnado. Por ello, pensamos que sigue siendo obligado el diseño de unidades didácticas (seleccionar temáticas, analizar el posible contenido a compartir con el alumnado, discutir sus problemas de aprendizaje, plantear una secuencia de enseñanza coherente con los análisis anteriores, elaborar los materiales y estrategias correspondientes...) y hacerlo con un modelo de planificación adecuado (Pro y Rodríguez 2010).

\section{Diseño del trabajo}

\section{Participantes}

Esta investigación se ha implementado en un CEIP de una población de Jaén, de unos 5000 habitantes que trabajaban principalmente en el sector agrícola. Se trata de un centro público que, desde sus comienzos, ha mostrado una gran preocupación por mejorar la práctica docente, participando en numerosos proyectos de innovación como Aprendizaje Basado en Números (ABN), Programa de Integración de las Competencias Básicas de Andalucía (PICBA), Plan de Convivencia, etc. Cuenta con 25 docentes, con 12 aulas de Educación Primaria (EP) y 6 para EI, con una ratio media de 20 alumnos/as por aula. Tenía matriculados a 314 alumnos/as; 100 eran de EI.

Las clases en la que se ha llevado a cabo la investigación han sido las de 5 años A y B. El número de alumnos/as entre las dos clases era de 33 (5 años $\mathrm{A}, 17$ y 5 años $\mathrm{B}, 16)$. Todos han participado; por tanto, se trata de un muestreo incidental en el que no se ha producido ninguna selección previa por el investigador. No obstante, a lo largo de la experiencia, se produjo una baja, ajena al trabajo realizado, con lo que la muestra final fue de 32 participantes.

Ambas clases presentaban un nivel muy adecuado en cuanto a la consecución de los objetivos didácticos y mostraban interés por aprender. No obstante, había dos niños que no eran capaces de seguir el ritmo normal de la clase, uno por presentar síntomas de inmadurez general -aún sin diagnosticar- y otro con NEE. La mayoría leía de forma comprensiva

1 Disponible en: https://www.fondation-lamap.org/

2 Disponible en: http://www.aecosan.msssi.gob.es/AECOSAN/docs/documentos/nutricion/educanaos/profesores_escuela_activa.pdf

3 Disponible en: https://cordis.europa.eu/project/rcn/78779/brief/es 
palabras y frases sencillas y empezaba a expresarse por escrito, aunque obviamente con limitaciones. El clima de convivencia era adecuado y respetaban las normas establecidas. Las familias, en general, eran participativas en todas las actividades para las que se solicitaba su colaboración.

\section{Propuesta de enseñanza}

Es difícil justificar la planificación de una unidad didáctica, sobre todo, si ésta se va reconstruyendo a partir de las intervenciones del alumnado. No obstante, vamos a centrarnos en varios elementos que consideramos relevantes en un proceso de estas características.

\section{Contextualización de la UD}

La propuesta se enmarca principalmente en el Área del Conocimiento del Entorno. No obstante, el carácter globalizador de esta etapa hace obligada la inclusión del Área de Conocimiento de sí mismo y autonomía personal y del Área del Lenguaje y Comunicación. En relación con el primero, por el progresivo descubrimiento de sus capacidades y limitaciones en su interacción reflexiva con el medio. Respecto al segundo, en su doble faceta: la adquisición de destrezas comunicativas (comprensión y uso del vocabulario adecuado, aumento de la compresión lectora y expresión escrita...) y la capacidad de trabajar de forma colaborativa, argumentando y respetando las opiniones de otros.

Si revisamos los objetivos de la etapa (BOJA 2008, pp. 24-25) creemos que, con nuestra propuesta, se puede contribuir a:

«reconocer e identificar sentimientos y emociones [...], saber comunicarlos a los demás reconociendo y respetando a los de otros»; «avanzar en la adquisición de hábitos y actitudes saludables»; «desarrollar capacidades de iniciativas [...] para contribuir a dotar de intencionalidad su acción, a resolver problemas habituales de la vida cotidiana y aumentar el sentimiento de autoconfianza»; «descubrir el placer de colaborar con los iguales, ir conociendo y respetando las normas del grupo y adquirir las actitudes y hábitos propios de la vida en un grupo social más amplio».

\section{Contenidos de la UD}

La temática de la electricidad, los aparatos eléctricos, los circuitos... está cercana al alumnado de estas edades. Para ellos, estos fenómenos tienen un cierto componente mágico, la motivación por construir algo que hace algo, la explicación de cómo funcionan sus juguetes... lo que les dota de un atractivo natural. Sin embargo, muchas veces no son conscientes de la peligrosidad que tiene, si no se toman las medidas para evitarla, ni del impacto ambiental que produce un uso inadecuado de los mismos. En cualquier caso, no pretendemos obviamente agotar el tema. Por ello, en la Figura 1 hemos representado los conocimientos declarativos que hemos pretendido compartir con el alumnado. 


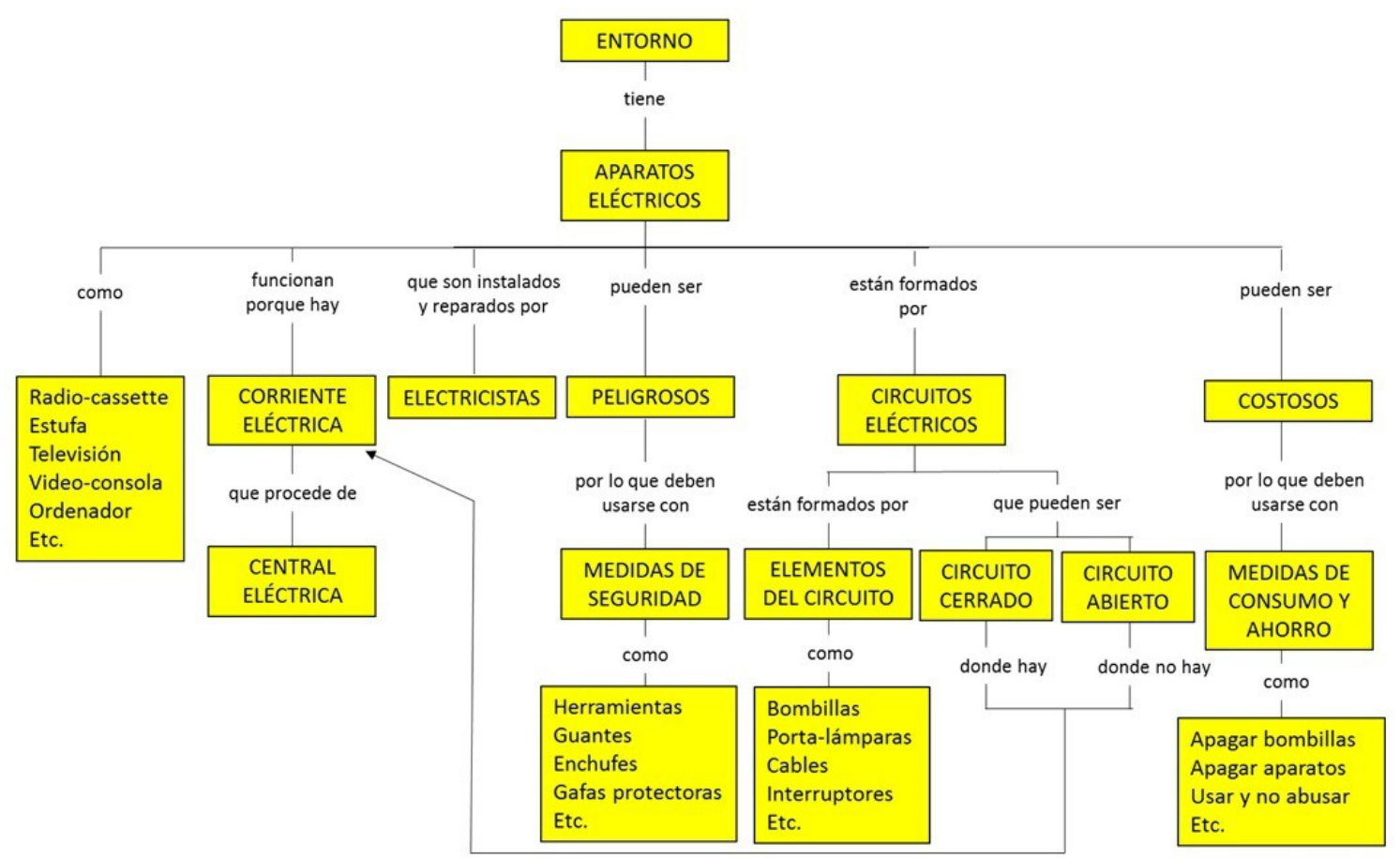

Figura 1. Contenidos de la Unidad Didáctica

No obstante, en una propuesta como la nuestra, juegan un papel fundamental otros conocimientos de carácter procedimental o actitudinal. Algunos se recogen en la Tabla 1.

Tabla 1. Contenidos incluidos en nuestra propuesta.

\begin{tabular}{|c|c|}
\hline Contenidos procedimentales & os actitudinales \\
\hline $\begin{array}{l}\text { - Observación. } \\
\text { - Identificación de objetos y reconocimiento de } \\
\text { algunas de sus propiedades. } \\
\text { - Uso del destornillador. } \\
\text { - Realización de un montaje: circuito eléctrico simple } \\
\text { con una bombilla con su portalámparas, unos cables, } \\
\text { una pila y un interruptor. } \\
\text { - Representación simbólica de elementos de los } \\
\text { circuitos. } \\
\text { - Búsqueda de información sobre temas relacionados } \\
\text { con los circuitos eléctricos. } \\
\text { - Uso de cámara fotográfica o de la cámara de un } \\
\text { móvil. }\end{array}$ & $\begin{array}{l}\text { - Rigor y precisión en la descripción de } \\
\text { observaciones. } \\
\text { - Valoración de la labor de los electricistas en la vida } \\
\text { cotidiana. } \\
\text { - Valoración de la importancia de las medidas de } \\
\text { seguridad con los aparatos eléctricos. } \\
\text { - Valoración de medidas de consumo racional de } \\
\text { energía eléctrica. } \\
\text { - Actitud científica en la realización de montajes. } \\
\text { - Curiosidad ante los hechos cotidianos. } \\
\text { - Actitud participativa y de respeto en los debates. } \\
\text { - Asombro y emociones con los logros. }\end{array}$ \\
\hline
\end{tabular}

\section{Problemas de aprendizaje}

Los alumnos de estas edades tienen algunos logros que debemos aprovechar; algunos de carácter cognoscitivo o manipulativo, por ejemplo, no suelen tener dificultad para identificar los aparatos eléctricos o para conectar los elementos de un circuito simple (Pro 2008). Pero, sobre todo, tienen tres motores muy útiles para aprender: la curiosidad, la capacidad de observación e imitación, y la actitud exploratoria.

Aunque sean escasas, hay que partir de las creencias y experiencias del alumnado. En algunos trabajos se han identificado ideas alternativas (Shepardson y Moje 1994, Solomonidou y Kakana 2000, Glauert 2009, Kada y Ravanis 2016): del modelo de corriente eléctrica, del papel de los elementos (especialmente de la batería o fuente), en las predicciones de comportamiento de circuitos o sobre el consumo. Según Hickling y Wellman (2001) hay 
razones por las que los niños de estas edades pueden tener ya ideas alternativas: experiencias sensoriales, confusiones terminológicas en el lenguaje cotidiano, concepciones culturales, relaciones con iguales... A estas concepciones podríamos añadirles otras características de carácter psicoevolutivo (Piaget e Inhelder 2000): condicionantes derivados de un egocentrismo intelectual, dotación cualidades humanas a los objetos (animismo), pensamiento localizado en hechos aislados y sin tener en cuenta el conjunto, no ajustan sus observaciones a lo que ven sino a lo que quieren ver...

Pero, sin duda, las mayores limitaciones provienen de la comprensión lectora y de la comunicación oral y, sobre todo, escrita. Precisamente la iniciación o el aprendizaje de la lectoescritura es uno de los objetivos de esta etapa educativa.

\section{Secuencia de enseñanza}

Nuestra secuencia de enseñanza está organizada por sesiones. En casi todas, se parte de una Asamblea, una sesión del gran grupo en la que se revisan las tareas realizadas en casa, se realizan puestas en común de trabajos realizados o se debate sobre una temática que previamente se ha trabajado en el aula. Luego, proponemos un aspecto fundamental de la forma en la que trabajamos: es lo que llamamos Situación problemática, en la que la maestra, previa contextualización, modelización e interacción reflexiva con los estudiantes, plantea un reto o tarea a realizar individual o colectivamente. Otros tipos de actividades son las que deben realizar los niños para dar respuesta al reto planteado; éstas pueden realizarse en clase, sólo o con otros compañeros, y en casa con sus padres.

Hemos tratado de describir de forma sintetizada las sesiones en la Tabla 2, aunque algunos materiales pueden encontrarse en http://agrega.juntadeandalucia.es/visualizar/es/esan_2015111812_9091851/null.

Tabla 2. Secuencia de enseñanza de «Jugando con la electricidad».

\begin{tabular}{|c|c|c|}
\hline Sesión & Descripción & $\begin{array}{c}\text { Tipo } \\
\text { actividad }\end{array}$ \\
\hline \multirow[t]{3}{*}{$1^{\mathrm{a}}$} & $\begin{array}{l}\text { Tarea inicial: Lectura del cuento «Bombillín, una bombilla feliz cuando ahorra } \\
\text { energía». La profesora plantea preguntas sobre el contenido del cuento: qué son las } \\
\text { bombillas, para qué sirven, cómo y por qué se encienden, qué tipos de bombillas } \\
\text { conocen, por qué hay que cuidar el consumo, qué se debe hacer cuando una } \\
\text { bombilla no funciona... Los alumnos van expresando sus respuestas al grupo, sin } \\
\text { ser valoradas por la maestra. Esta se limita a hacer una síntesis de todas las ideas } \\
\text { que han aparecido en la asamblea. }\end{array}$ & Asamblea \\
\hline & $\begin{array}{l}\text { La maestra lanza el reto de reconocer los aparatos eléctricos del aula. Los alumnos } \\
\text { deben responder de forma ordenada y respetando el uso de la palabra. La maestra } \\
\text { debe preguntar cómo justifican el por qué lo son o no lo son. }\end{array}$ & $\begin{array}{c}\text { Situación } \\
\text { problemática }\end{array}$ \\
\hline & $\begin{array}{l}\text { Luego los alumnos deben realizar la Tarea 1: identificar los aparatos eléctricos (se } \\
\text { les presenta } 8 \text { objetos: radiocasete, estufa, televisión, plastilina, pizarra, vídeo- } \\
\text { consola, lápices de colores y ordenador). }\end{array}$ & $\begin{array}{l}\text { Trabajo } \\
\text { individual }\end{array}$ \\
\hline \multicolumn{3}{|c|}{$\begin{array}{l}\text { Tarea } 2 \text { para casa: Con la ayuda de los padres, realizar fotografías de aparatos eléctricos (podían sustituirse por } \\
\text { fotos de revistas o de catálogos de juguetes). Luego hacer un mural con ellas. }\end{array}$} \\
\hline \multirow[t]{3}{*}{$2^{a}$} & $\begin{array}{l}\text { Los alumnos muestran sus fotografías o recortes de aparatos eléctricos. Se comenta } \\
\text { para qué se utiliza cada uno de los que aparecen. } \\
\text { Se monta el Rincón de Electricidad en el que se van a depositar algunos de los } \\
\text { trabajos realizados en sus casas; en este caso, murales con algunas fotografías o } \\
\text { recortes. }\end{array}$ & Asamblea \\
\hline & $\begin{array}{l}\text { Con la ayuda de la pizarra digital, pregunta de dónde viene la corriente eléctrica. } \\
\text { Los alumnos van respondiendo las cuestiones que les va planteando la maestra } \\
\text { hasta que una señala una central próxima (Procesos Ecológicos Vilches) al colegio. }\end{array}$ & $\begin{array}{c}\text { Situación } \\
\text { problemática }\end{array}$ \\
\hline & $\begin{array}{l}\text { Salida al patio para observar la central, las torres eléctricas, los cables, la estación de } \\
\text { distribución, los cuadros eléctricos del colegio y los tubos con cables que llegan a } \\
\text { las aulas. }\end{array}$ & $\begin{array}{c}\text { Salida al } \\
\text { patio }\end{array}$ \\
\hline
\end{tabular}




\begin{tabular}{|c|c|c|}
\hline & $\begin{array}{l}\text { Los alumnos deben resolver la Tarea 3: A partir de una representación en papel de } \\
\text { las cercanías del colegio, identificar diferentes lugares (el colegio, la plaza de la } \\
\text { Romería, parque infantil... y la central) y señalar el camino desde la central al } \\
\text { colegio. Señalar con un dedo el camino a recorrer, luego con un lápiz y, por último, } \\
\text { con un rotulador. }\end{array}$ & $\begin{array}{l}\text { Trabajo } \\
\text { colectivo }\end{array}$ \\
\hline \multirow[t]{3}{*}{$3^{a}$} & $\begin{array}{l}\text { Los alumnos deben identificar algunos electricistas del pueblo. Debaten qué hacen } \\
\text { y la peligrosidad de su profesión. Una alumna comenta un accidente que sufrió uno } \\
\text { de ellos y la maestra explica los peligros y las precauciones de la profesión. }\end{array}$ & Asamblea \\
\hline & $\begin{array}{l}\text { La maestra trae algunas herramientas, utensilios de seguridad y materiales que } \\
\text { utilizan los electricistas en su profesión. Se tocan, se discute su utilidad y, sobre } \\
\text { todo, se valora la importancia que tienen para evitar accidentes. }\end{array}$ & $\begin{array}{c}\text { Situación } \\
\text { problemática }\end{array}$ \\
\hline & $\begin{array}{l}\text { Los alumnos deben resolver la Tarea } 4: \text { señalar los objetos que utilizan los } \\
\text { electricistas para protegerse de los peligros de su profesión (herramientas, guantes, } \\
\text { enchufe, gafas protectoras, tijeras y potenciómetro) }\end{array}$ & $\begin{array}{l}\text { Trabajo } \\
\text { individual }\end{array}$ \\
\hline \multicolumn{3}{|c|}{$\begin{array}{l}\text { Tarea } 5 \text { para casa: Con la ayuda de los padres, buscar información sobre medidas de seguridad para el uso de } \\
\text { los aparatos eléctricos. Realizar una lista de medidas. }\end{array}$} \\
\hline \multirow[t]{3}{*}{$4^{a}$} & $\begin{array}{l}\text { Se hace una puesta en común con las medidas de seguridad buscadas por los } \\
\text { estudiantes para el uso de aparatos eléctricos y, en general, de la electricidad. }\end{array}$ & Asamblea \\
\hline & $\begin{array}{l}\text { La maestra lanza la idea de señalar aquellos objetos del aula en los que se deben } \\
\text { tener en cuenta las medidas de seguridad. }\end{array}$ & $\begin{array}{c}\text { Situación } \\
\text { problemática }\end{array}$ \\
\hline & $\begin{array}{l}\text { Los alumnos deben resolver la Tarea 6: colorear unos pictogramas y colocarlos, por } \\
\text { grupos, en aquellos puntos del aula en lo que debe tenerse cuidado con la } \\
\text { electricidad (enchufes, interruptores, aparatos eléctricos...). }\end{array}$ & $\begin{array}{l}\text { Trabajo } \\
\text { individual y } \\
\text { colectivo }\end{array}$ \\
\hline \multirow[t]{2}{*}{$5^{a}$} & $\begin{array}{l}\text { La maestra narra el cuento «La linterna que se perdió en el campo». A partir del } \\
\text { mismo, identifica, con sus estudiantes, los elementos de un circuito: las bombillas y } \\
\text { los portalámparas, los cables, los interruptores y las pilas. Debate con los } \\
\text { alumnos/as sobre los tipos de pilas, su utilidad, las medidas de seguridad, los } \\
\text { lugares para depositarlas una vez gastadas... }\end{array}$ & Asamblea \\
\hline & $\begin{array}{l}\text { Los alumnos/as deben realizar la Tarea 7: unir con flechas dibujos de diferentes } \\
\text { elementos (bombilla, interruptor, porta-lámpara, cable y pilas) y sus nombres. }\end{array}$ & $\begin{array}{c}\text { Trabajo } \\
\text { individual }\end{array}$ \\
\hline \multicolumn{3}{|c|}{$\begin{array}{l}\text { Tarea } 8 \text { para casa: Identificar las vocales de las palabras «bombillas», "pila» y «cables». Buscar en casa cinco } \\
\text { objetos que empiecen con las letras b (bombilla), p (pila) y c (cable). Decir qué vocal es la que más se repite y } \\
\text { realizar una lista con las palabras encontradas. }\end{array}$} \\
\hline \multirow[t]{3}{*}{$6^{a}$} & $\begin{array}{l}\text { La maestra sube algunas tareas de casa al Rincón de Lengua; las comenta, } \\
\text { preguntando a sus autores sobre la lista que han aportado. }\end{array}$ & Asamblea \\
\hline & $\begin{array}{l}\text { La maestra narra el cuento «Usando claves para hablar con circuitos» e introduce la } \\
\text { conveniencia de usar símbolos para representar los elementos de un circuito. A } \\
\text { medida que se simbolizan, los estudiantes los dibujan en unos círculos de papel. }\end{array}$ & $\begin{array}{c}\text { Situación } \\
\text { problemática }\end{array}$ \\
\hline & $\begin{array}{l}\text { Se juega a montar un «circuito humano»: en grupos, los alumnos, llevando unos } \\
\text { elementos en cartulina en el pecho, se unen para representar un circuito eléctrico. } \\
\text { Se presta especial atención al vocabulario, más técnico y preciso. }\end{array}$ & $\begin{array}{c}\text { Juego de } \\
\text { simulación }\end{array}$ \\
\hline \multicolumn{3}{|c|}{$\begin{array}{l}\text { Tarea } 9 \text { para casa: Con la ayuda de los padres, hacer un listado de los aparatos eléctricos de la cocina y del } \\
\text { cuarto de baño. Luego responde: ¿cuántos aparatos eléctricos hay en las dos habitaciones? }\end{array}$} \\
\hline \multirow[t]{3}{*}{$7^{a}$} & $\begin{array}{l}\text { Se hace una puesta en común de los murales elaborados por los alumnos. Ellos } \\
\text { explican los elementos que han fotografiado. }\end{array}$ & Asamblea \\
\hline & $\begin{array}{l}\text { La maestra repasa brevemente los elementos de un circuito y explica cómo } \\
\text { debemos conectarlos, usando un destornillador: bombilla y porta-lámpara y cables } \\
\text { con porta-lámparas. Luego invita a hacerlo a algunos para modelizar la siguiente } \\
\text { tarea. }\end{array}$ & $\begin{array}{c}\text { Situación } \\
\text { problemática }\end{array}$ \\
\hline & $\begin{array}{l}\text { Los alumnos/as deben realizar la Tarea 10a: Usar el destornillador para atornillar } \\
\text { los cables a los portalámparas (están encajados en la rosca; han sido ligeramente } \\
\text { desatornillados) y posteriormente ajustarle una bombilla. Hay una persona en el } \\
\text { aula para ayudar a la maestra con los estudiantes con dificultades. }\end{array}$ & $\begin{array}{l}\text { Trabajo } \\
\text { individual }\end{array}$ \\
\hline \multirow[t]{2}{*}{$8^{a}$} & $\begin{array}{l}\text { Se revisa lo realizado el día anterior: nombre de los elementos, ajuste de cables al } \\
\text { portalámparas, enroscar la bombilla en el porta-lámpara... }\end{array}$ & Asamblea \\
\hline & $\begin{array}{l}\text { La maestra explica cómo realizar un circuito simple con una pila, una bombilla y un } \\
\text { interruptor y plantea el reto de que se hagan circuitos con el material que tienen } \\
\text { delante. }\end{array}$ & $\begin{array}{c}\text { Situación } \\
\text { problemática }\end{array}$ \\
\hline
\end{tabular}




\begin{tabular}{|c|c|c|}
\hline & $\begin{array}{l}\text { Los alumnos/as deben realizar la Tarea 10b: Usando el destornillador, realizar un } \\
\text { montaje de un circuito simple con una bombilla. Hay una persona en el aula para } \\
\text { ayudar a la maestra con los estudiantes con dificultades. }\end{array}$ & $\begin{array}{c}\text { Trabajo } \\
\text { individual }\end{array}$ \\
\hline & Explica el papel del interruptor en un circuito cerrado y abierto, con un flexo. & $\begin{array}{c}\text { Situación } \\
\text { problemática }\end{array}$ \\
\hline & $\begin{array}{l}\text { Los alumnos/as deben realizar la Tarea 10c: Accionando el interruptor, comprobar } \\
\text { que el circuito funciona correctamente. Mostrar el circuito completo a los demás, } \\
\text { explicando el funcionamiento. La maestra resalta el trabajo realizado y los incorpora } \\
\text { al Rincón. }\end{array}$ & $\begin{array}{l}\text { Trabajo } \\
\text { individual y } \\
\text { Asamblea }\end{array}$ \\
\hline \multicolumn{3}{|c|}{$\begin{array}{l}\text { Tarea } 11 \text { para casa: Buscar en vuestra casa cinco aparatos que tengan circuitos abiertos y otros que los tenga } \\
\text { cerrados. Hacer una lista de ambos. }\end{array}$} \\
\hline \multirow[t]{3}{*}{$9^{\mathrm{a}}$} & $\begin{array}{l}\text { La maestra pone un episodio de «Enermanos» para sensibilizar al alumnado sobre } \\
\text { la importancia de un consumo racional de energía y los beneficios de utilizar } \\
\text { medidas de ahorro. Mediante cuestiones indaga en el contenido (¿cómo se llamaban } \\
\text { los personajes?; ¿qué problema trataban de resolver?; ¿cómo lo hacían?; ¿qué } \\
\text { hubieras hecho tú?...). }\end{array}$ & Asamblea \\
\hline & $\begin{array}{l}\text { Una alumna muestra preocupación por el cambio climático. La maestra lanza el } \\
\text { reto de qué podrían hacer para ahorrar energía eléctrica en el aula y así contribuir a } \\
\text { mejorar nuestro planeta. }\end{array}$ & $\begin{array}{c}\text { Situación } \\
\text { problemática }\end{array}$ \\
\hline & $\begin{array}{l}\text { Los alumnos, en grupos, deben realizar la Tarea 12: Realizar un listado de acciones } \\
\text { para usar adecuadamente energía eléctrica, realizar un consumo aceptable e, incluso, } \\
\text { para ahorrarla. Luego a partir del debate, se hace un compromiso de obligado } \\
\text { cumplimiento. }\end{array}$ & $\begin{array}{l}\text { Trabajo } \\
\text { individual y } \\
\text { colectivo }\end{array}$ \\
\hline $10^{\mathrm{a}}$ & $\begin{array}{l}\text { La maestra muestra cómo realizar un circuito eléctrico con plastilina, tanto la } \\
\text { conductora como la aislante: https://www.ted.com/talks/annmarie_thomas_ } \\
\text { squishy_circuits } \\
\text { Se realiza Tarea 13: cada familiar realiza un circuito (plastilina, cables, bombillas led, } \\
\text { pila) con su hijo/a. Se decora la plastilina (caras, flores, animales, barcos...). }\end{array}$ & $\begin{array}{l}\text { Asamblea y } \\
\text { trabajo con } \\
\text { los padres en } \\
\text { el aula }\end{array}$ \\
\hline $11^{\mathrm{a}}$ & $\begin{array}{l}\text { Por último, con la pizarra digital, la maestra entra en la plataforma Averroes de la } \\
\text { Junta de Andalucía (Tarea 14). Se repasa lo trabajado en sesiones anteriores y se } \\
\text { realizan ejercicios interactivos. }\end{array}$ & $\begin{array}{c}\text { Trabajo } \\
\text { colectivo }\end{array}$ \\
\hline $12^{\mathrm{a}}$ & $\begin{array}{l}\text { Bombillín -compañera disfrazada de bombilla- realiza preguntas a cada alumno/a } \\
\text { sobre el tema (Tarea 15). Pregunta central: ¿Qué has aprendido en este Proyecto? } \\
\text { ¿Qué es lo que más te ha gustado? }\end{array}$ & $\begin{array}{l}\text { Actividad } \\
\text { evaluación }\end{array}$ \\
\hline
\end{tabular}

La propuesta ha sido trabajada paralelamente en los dos grupos. Realizamos numerosas reuniones de coordinación para que no hubiera diferencias en su desarrollo (ni en la labor de las docentes ni en el trabajo del alumnado); por ello, tratamos la información conjuntamente.

\section{Instrumentos de recogida de información}

Siempre es compleja la recogida de información con niños/as de estas edades por los problemas de comunicación -sobre todo, escrita- a pesar de la situación favorable de nuestros participantes. No obstante, en este trabajo, hemos utilizado: las intervenciones y los debates generados en las asambleas; las producciones de los alumnos/as en los trabajos individuales; las tareas realizadas en casa con sus familiares; y las respuestas en la sesión de evaluación. Toda la información recogida se ha ratificado con el diario de las dos maestras que han intervenido en la experiencia, siguiendo las orientaciones realizadas al respecto (Porlán y Martín 2000, Pro 2011): denominación de actividad, contenidos trabajados, organización, temporalización, preguntas de los estudiantes (en el planteamiento y desarrollo de la actividad), aspectos positivos y problemáticos (en relación con la motivación inicial, atención, clima de clase, relaciones personales, cooperación e implicación), incidencias y observaciones.

\section{Análisis de resultados}

Vamos a distinguir los resultados de las tareas: la inicial, las realizadas en clase, las que hicieron en casa (en muchos casos, con ayuda de adultos) y la desarrollada en la sesión de evaluación. 


\section{Tarea inicial}

Para comenzar este proyecto, se narra un cuento en ambas clases sobre «Bombillín, una bombilla feliz cuando ahorra energía»; también las maestras trajeron diferentes bombillas. Con ello pretendíamos motivar al alumnado por el tema y poner de manifiesto sus ideas previas. Los/as alumnos/as mostraron gran interés por la temática y participaron animadamente en un pequeño debate al final del cuento.

Queremos destacar algunos comentarios que surgieron como: «las bombillas se cuelgan de la pared y funcionan», «en las pilas está metida la electricidad», «los albañiles meten los cables de la luz», «la electricidad está en el enchufe», «los rayos tienen electricidad»... Así mismo, algunos no sabían diferenciar una bombilla de bajo consumo de una tradicional; llamaban «botón» al interruptor; hablaban indistintamente de bombillas o luz para referirse al elemento; utilizaban un modelo de fluido («la electricidad sale de la pila») para referirse a la corriente eléctrica; asociaban la electricidad a conceptos mágicos, malditos o venenosos... Además, aparecieron temáticas transversales como el reciclado, el punto limpio, etc.

Podemos decir que el grupo tenía ideas y experiencias aprovechables, tal como han recogido otros trabajos (Solomonidou y Kakana 2000, Glaubert 2009, Kada y Ravanais 2016). Además, en nuestro caso, tenían facilidad en la comunicación oral y estaban iniciados en la expresión escrita.

\section{Tareas en clase}

En relación con la tarea 1 de la propuesta didáctica, se preguntaba al alumnado: «María quiere ordenar los aparatos eléctricos que hay en la clase y le vamos a ayudar. Para ello, tienes que hacer un círculo a los aparatos eléctricos que encontrarás más abajo». Casi todos los participantes (30/32) reconocieron todos los aparatos eléctricos que se le mostraban e, incluso, los etiquetaron con su nombre. Tan sólo dos -A5 y A7- no los reconocieron todos, posiblemente consecuencia de alguna distracción a la hora de realizar la tarea. Incluso, la mayoría señalaba otros que no aparecían: móvil, robot, pizarra digital...

Respecto a la tarea 3, se pedía al alumnado: «Hoy en clase hemos tenido un problemilla porque no se encienden las bombillas y María no sabe cómo arreglarlo... Averigua el camino que hace la luz desde la central hasta nuestro cole...». Para ello, tras la explicación, se les dio un dibujo esquemático de las proximidades al colegio e hicieron el recorrido con los tres procedimientos (dedo, lápiz y rotulador). Todos la han realizado adecuadamente, aunque usaron dos alternativas posibles: empezando por el colegio o por la central.

En relación con la tarea 4, se preguntaba: «María necesita que le ayudemos con las normas de seguridad de los electricistas. Haz un círculo a los tres objetos que utilizan cuando trabajan. Después los compararemos con los de nuestros compis de clase. Adelante...». La mayoría del grupo -26/32- reconocieron cuáles eran las herramientas o utensilios de seguridad para trabajar con los circuitos eléctricos. Solo 6 alumnos -A6, A20, A27, A28, A29 y A32cometieron un error, aunque acertaron los demás; éste curiosamente fue el mismo: el potenciómetro, aparato que probablemente desconocían.

En la tarea 6, se pedía al alumnado que colorearan unos pictogramas y los colocaran en los lugares del aula en los que debía tenerse cuidado con la electricidad. Aunque algunos tuvieron algún problema al colorear (se salían de los límites del trazado de los dibujos), ninguno tuvo dificultades para identificar los objetos problemáticos (enchufes, interruptores, aparatos, bombillas...) y estuvieron muy motivados -a veces demasiado, con el consiguiente conflictopara colocar los pictogramas. Tampoco encontraron impedimentos para asociar dibujos y 
denominaciones con los elementos en la tarea 7; de hecho, fue realizada adecuadamente por casi todos los participantes, menos uno -A26- que no identificó la pila.

En la tarea 10, se señalaba: «Prestar atención a la maestra porque va a montar un circuito eléctrico. Lo va a hacer en tres pasos. Cuando termines cada uno de ellos, por favor, avisa... Después, coge los elementos del circuito que tienes en tu mesa y únelos a ver qué pasa...». Esta actividad ha sido la más divertida para el alumnado: ellos iban a montar un circuito eléctrico con una pila, una bombilla, un portalámparas, unos cables y un interruptor. Para organizar mejor la actividad, la dividimos en tres partes.

En la primera, tras una breve explicación por la maestra, nos sorprendió la facilidad con la que gran parte del grupo manejaban el destornillador; no obstante, tuvimos que ayudar a una tercera parte de ambas clases (normalmente porque soltaron algún tornillo del portalámparas). Pero, en general, como hemos dicho, en un clima de implicación y motivación extraordinarios.

En la segunda y la tercera, fue aún más impactante: la soltura para enroscar las bombillas en sus portalámparas; la habilidad para enganchar la pila, el interruptor o los cables; el desparpajo para abrir y cerrar el circuito... El entusiasmo era generalizado y uno de los alumnos -en un clima de agitación emocional colectiva porque todos los circuitos funcionaban- gritó: «iEs verdad! ¡Funciona! ¡Se me ha encendido!...». Pusimos a oscuras la clase para apreciarse mejor el efecto. El canto final fue impactante.

Respecto a la tarea 12, planteábamos: «María quiere que no hagamos un mal uso de la energía eléctrica. Para ello, tenéis que proponer tres medidas en grupo. Eso sí: debéis tener en cuenta que después es obligatorio cumplir lo que digáis». La acción que propusieron todos era «Apagar las luces del aula» con todas sus modalidades: cuando salimos, por la mañana, los fines de semana, mientras estamos en el recreo... Otra acción también muy mencionada se refería a «Apagar...»y señalaban «el ordenador», «las videoconsolas», «el televisor», «los móviles», «el aire acondicionado», «la pizarra»... Hubo algunas respuestas ingeniosas: «Quitar las pilas cuando no se usa [se referían a una linterna]»; «Cortar el cuadro general del colegio»; «No poner...» y señalaban algunos de los aparatos ya mencionados. Incluso, aparecieron algunas medidas de ahorro: «Cambiar los tubos de la luz por otros que gasten menos»; «No encender todas las luces de la clase» o «Abrir las ventanas para que entre la luz».

En el debate, se eligieron tres para que realmente se cumplieran y se pudieran controlar por todos: «Apagar las luces del aula»; «Apagar todos los aparatos al terminar el día» y «Desenchufar todos los aparatos cuando no se usen». Y se hizo un documento para firmarlo todos. Luego se colocó en el Rincón.

En relación con la tarea 13, cada familiar con su hijo/a realizó circuitos eléctricos utilizando la plastilina, los cables, una bombilla de led y una pila. Empezaron realizando uno con dos masas conductoras que no estaban unidas, seguidamente usaron la aislante para ponerla entre medias... Luego, cada alumno/a realizó un circuito utilizando una figura distinta (caras, flores, animales, barcos...). Algo curioso: los alumnos/as mostraron más seguridad que sus familiares. La actividad fue muy gratificante tanto para las familias como para el alumnado.

Por último, en la tarea 14, el alumnado trabajó, de forma interactiva, con la pizarra digital en la plataforma Averroes. Evidentemente, fue muy motivante para los niños y niñas de ambos grupos y la mayoría respondieron sin aparente dificultad a lo que les planteaban las maestras. Si hubiera que mencionar algún obstáculo, éste sería de tipo técnico: los estudiantes apretaban mucho o soltaban el dedo a la hora interactuar con los juegos. Los que tuvieron «más dudas» fueron A13, A21 y A32. 


\section{Tareas realizadas en casa}

También hemos analizado las actividades realizadas en casa. Debido a la singularidad de las tareas propuestas, nos hemos centrado en valorarlas y el grado de satisfacción de la misma.

La tarea 2 de la propuesta didáctica, decía: «Con papá y mamá, debéis realizar fotografías a aparatos o máquinas que haya en vuestra casa. Si no podéis, buscarlos y recortarlos en una revista, catálogo de juguetes, etc.». Realizaron las fotografías 31/32, ya que A32 no hizo ni ésta ni las demás tareas de casa. Aunque casi la totalidad acertaron, la calidad de las imágenes fue más desigual; no obstante, se notó la ayuda familiar. En cuanto al grado de satisfacción, a más del 90\% le ha gustado «mucho»; a dos -A21 y A22- sólo «regular».

En la tarea 5, se planteaba: «Con ayuda de tu familia, busca información en Internet sobre las normas de seguridad con los circuitos eléctricos. A continuación, escribidlas en una hoja». Esta actividad fue realizada por 29/32 participantes. Algunas de las propuestas realizadas fueron: «ver alguna serie de dibujos que trate el tema»; «poner letreros en los enchufes»; «aprender a cambiar bombillas en casa con ayuda de un familiar»; «cortar la electricidad desde el cuadro general»; «aprender a usar DVD, TV, micro-ondas... con la ayuda de un mayor»; «no tocar los aparatos eléctricos si estás mojado»; «no tocar cables en mal estado»... A 27/32 le gustó «mucho»; a 4/31 -en concreto A1, A3, A19 y A24- sólo «regular»; y A32 no la llegó a realizar.

En la tarea 8, se pedía al alumnado: «María quiere repasar algunas letras utilizando los circuitos eléctricos. Sigue las instrucciones de la tarea que te va a leer tu maestra». Puesto que estamos en EI, la propuesta tiene un carácter globalizador con contenidos de otras materias; en este caso, de lecto-escritura (en el centro se trabaja en esta etapa educativa). Hablando con la maestra, creía que se debía a algún despiste porque normalmente sabían realizarla. Un alumno -A21- no la realizó porque faltó a clase.

En relación a la tarea 7, se proponía: «María quiere que señaléis las vocales de bombillas, pila y cables». Luego debes buscar cinco objetos de vuestra casa que empiecen por las letras $b, p, c$. Tenéis que hacer una lista». Esta actividad la realizaron 30/32 participantes; no la hicieron A21 y A32. En esta actividad, 26/32 de los participantes la realizaron adecuadamente. El resto del alumnado dejaron algo sin responder: un participante -A28- una palabra y otros cuatro -A3, A14, A20 y A25- alguna vocal. A todos los participantes les ha gustado «mucho» la actividad. Muchos de ellos -a sugerencia de la maestra- escribieron previamente las palabras en distintas superficies (arena, vaho, papel, plastilina, etc.); probablemente esto justifique el éxito de la tarea.

Con respecto a la tarea 9, decíamos: «María quiere que contéis los aparatos eléctricos de casa. Para ello, suma los aparatos que veas en la cocina con los del cuarto de baño». Esta actividad la realizaron 28/32 participantes; de los otros, tres -A13, A15 y A31- no vinieron a clase por estar enfermos. En general, las respuestas respondieron a los aparatos que tenían en el listado, aunque es posible que no estuvieran localizados todos los aparatos. En cuanto al grado de satisfacción, volvió a ser mayoritario el «me ha gustado mucho» con 22/32, a 4/31 alumnos les gustó «regular» y a $2 / 31$ «poco». Sin duda, ésta fue la actividad peor valorada. Creemos que influyó el cansancio acumulado o una cierta incomprensión sobre lo que se les preguntaba (realizar una simple operación matemática).

En relación con la tarea 11, se planteaba: «María esta vez os necesita... Tenéis que buscar en vuestra casa si hay circuitos abiertos y circuitos cerrados. Para ello, debéis hacer una lista de cinco aparatos que tengan el circuito abierto y otros cinco que estén cerrados. Luego explicareis, con ayuda de vuestra maestra, por qué están abiertos o cerrados». Esta actividad era un poco más compleja que las anteriores. No obstante, 29/32 resolvieron bien esta 
actividad y, además, hicieron los listados, evidentemente con errores ortográficos y de expresión propios de la edad, aunque no fuera el fin último que se perseguía en este trabajo. Tres -A13, A21 y A32- sólo fueron capaces de encontrar circuitos cerrados (aunque hubiera numerosos abiertos: luces apagadas, aparatos sin funcionar...).

Aunque no ha sido objeto del trabajo la valoración de la experiencia por los padres, disponemos de esta información. En líneas generales los comentarios más utilizados por las familias han sido: «no quería parar de jugar», «estas actividades están siendo muy divertidas», «me están gustando mucho», «estamos aprendiendo en familia», «nos lo estamos pasando fenomenal...».

\section{Tarea final}

Como dijimos, realizamos una valoración final, a partir de las preguntas planteadas por un personaje creado al efecto. Al respecto, podemos decir que, más del $90 \%$ de los participantes, son capaces de:

- Identificar los aparatos eléctricos del entorno (clase, casa, colegio...).

- Identificar, representar y conocer la función de los elementos de un circuito eléctrico simple.

- Montar y hacer funcionar un circuito simple con una pila, un interruptor, un portalámparas, unos cables y una bombilla.

- Conocer algunas medidas de sostenibilidad relacionadas con el uso de los circuitos eléctricos.

- Escuchar y participar en las Asambleas y en las tareas colectivas, con respeto y tolerancia.

Entre el 80\% y el 90\%, son capaces de:

- Identificar una central para producir corriente eléctrica; unas torres eléctricas y unos cables para «trasportarla»; unas estaciones para su distribución y un contador en la entrada del colegio, a partir de una representación de un mapa.

- Conocer algunas normas de seguridad para el uso de los circuitos eléctricos.

- Usar el destornillador con cierta destreza.

- Diferenciar y reconocer circuitos abiertos y cerrados.

- Escribir palabras que empiecen por unas consonantes (b, p, c), identificar las vocales de unas palabras y realizar cálculos sencillos.

Entre las mayores dificultades, podemos citar que no son capaces de:

- Mantener un cierto orden y limpieza en la realización de las tareas de clase y entregar puntualmente las realizadas en casa.

- Usar el ratón en la pizarra digital.

- Utilizar un vocabulario adecuado en sus intervenciones y diferenciar términos que científicamente tienen diferentes significados (electricidad-corriente, energía-fuerza...).

- Tener un modelo adecuado de corriente eléctrica: creen que la electricidad es un fluido que sale de la pila o del enchufe y se mueve por los circuitos.

- A pesar de los avances, comprender todo lo que leen y, sobre todo, expresar lo que piensan por escrito. 


\section{Conclusiones}

Como hemos comentado anteriormente, el objetivo central de esta investigación ha sido el diseño de una propuesta de enseñanza sobre el Estudio de la Electricidad en EI, su puesta en práctica y la valoración de algunos de los efectos producidos respecto al aprendizaje del alumnado. Realizado el análisis de la información recogida, vamos a sintetizar algunas de las conclusiones en base a los resultados obtenidos.

En primer lugar, hemos de decir que el modelo de planificación utilizado nos ha permitido un diseño adecuado ya que hemos podido seleccionar contenidos científicamente relevantes, tener en cuenta logros y limitaciones de los estudiantes, construir conocimientos de diferentes tipologías (conceptuales, procedimentales y actitudinales), utilizar medios y recursos cercanos a los niños, y una evaluación integrada en el proceso de aprendizaje. Además, tiene la flexibilidad necesaria para la adaptación a las aportaciones, inquietudes e intereses de los estudiantes.

El desarrollo estructural de las tareas fue el previsto inicialmente, con algunas matizaciones que surgieron por la implicación del alumnado, y en líneas generales podemos calificarlo como bueno. Hemos pretendido, desde el principio, que el alumnado se sintiera el protagonista de todo el proceso. Incluso, en los momentos en los que se planteaban las situaciones problemáticas, hemos intentado -y creemos que conseguido- que los niños hicieran suyo los problemas y retos que les planteábamos, tanto en las tareas más «prácticas» (realización de experimentos o fotografías) como en las de carácter más «teóricas» (cuestiones de asociación o búsqueda de información).

Aunque no hemos pretendido que investigaran, por lo menos hemos intentado que experimentaran, paso previo para llevar a cabo experiencias de mayor complejidad cognitiva. En este sentido, creemos que los niños tienen un potencial bastante desaprovechado actualmente en algunas aulas, en las que la sobreprotección o los miedos parece que se anteponen a cualquier planteamiento que pueda generar alguna inseguridad.

También nos parece importante la sensibilización infantil sobre los problemas de desarrollo sostenible, la realización de acciones adecuadas de consumo y ahorro (de agua, de energía...). $\mathrm{Y}$, además, trabajarlo de manera que se vean como parte de la solución de los problemas medioambientales. No como si fueran causados por otros y fueran otros los que debieran encontrar la solución. Se pueden hacer cosas desde las edades más tempranas, obviamente adecuándolas a sus posibilidades educativas.

Nos ha parecido muy interesante la participación de las madres y padres de los estudiantes. Aunque las tareas de casa han sido, en algunos casos, una caja negra respecto al desarrollo, al papel de los progenitores o al tiempo y dedicación que han supuesto, existe un cierto consenso en la valoración positiva de las actividades realizadas (sobre todo, la realización de fotografías). En cuanto a la búsqueda de información (sobre medidas de seguridad), detectamos que la utilizada provenía de páginas que no son adecuadas para estas edades. Creemos que deberíamos orientar más a los padres en el uso de Internet con sus hijos.

En cuanto a los efectos producidos por la propuesta, hemos de decir que no tienen problemas con la identificación de aparatos eléctricos; son capaces de identificar, nombrar y representar elementos como bombillas, portalámparas, interruptores, cables y pilas; utilizan el destornillador con cierta solvencia; pueden montar y accionar un circuito eléctrico simple; se han producido avances en el aprendizaje de la lecto-escritura y en el cálculo; han mejorado su vocabulario, su comunicación y su actitud ante el trabajo cooperativo. Obviamente, en este momento, no sabemos si estos aprendizajes serán o no duraderos, pero tenemos la intención de valorar la situación trascurrido un tiempo significativo. 
Por último, hemos de recalcar que el alumnado y sus maestras han disfrutado -y mucho- en el desarrollo de esta experiencia. Pero, además de divertirse, los primeros han aprendido, han percibido que era útil lo que hacían, se han implicado, se han asombrado, han aplaudido cuando estaban satisfechos, no sólo participaban sino que pedían participar, han generado una complicidad envidiable en el aula... En definitiva, hemos conseguido que hablaran de ciencias, una vez que acababa la clase de los circuitos.

En definitiva, creemos que ha sido una experiencia muy positiva y enriquecedora para toda la comunidad educativa a pesar del trabajo y el esfuerzo que conlleva diseñar e implementar propuestas didácticas con alumnado distinto al tuyo. Creemos honestamente que hemos favorecido aprendizajes cooperativos y duraderos (aunque no tengamos constancia experimental de ello) para los alumnos, probablemente porque se han sentido protagonistas de sus aprendizajes. De hecho, con esta experiencia queremos despertar la curiosidad o motivación a otros docentes, aunque no tratamos de dar recetas, pero sí aportar un punto de partida para empezar algo sobre lo que discutir y, por supuesto, criticar; pero si nuestro trabajo sirve a algún maestro para reflexionar sobre lo que hacen o pueden hacer, nos daremos por satisfechos.

\section{Referencias bibliográficas}

Benarroch A. (2012) La investigación en Didáctica de las Ciencias Experimentales en las etapas educativas de Infantil y Primaria. En A. M. Abril, A. Quesada (Eds.), XXIV Encuentros de Didáctica de las Ciencias Experimentales (pp. 32-52). Baeza: Ser. Pub. Univ. Jaén.

BOJA (2008) Orden de 5 de agosto de 2008, por la que se desarrolla el Currículo correspondiente a la Educación Infantil en Andalucía. BOJA (Boletín Oficial de la Junta de Andalucia), 169, 16 de agosto de 2008, 17-53.

Cantó J., de Pro A., Solbes J. (2016) ¿Qué ciencias se enseñan y cómo se hace en las aulas de educación infantil? La visión de los maestros en formación inicial. Enseñanza de las Ciencias 34(3), 25-50.

Cobe, K. (2015) Teaching Science during the Early Chilhood Years. National Geographic Learning/Cenage.

Duit R., Treagust D. F. (1995) Students' conceptions and constructivist teaching approaches. En B. J. Fraser, H. J. Walberg (Eds.), Improving science education. (pp. 46-69). Chicago: The University of Chicago Press.

Eshach H, Fried M. N. (2005) Should science be taught in early childhood? Journal of Science Education and Technology 14(3), 315-336.

Fleer M. (2015) A cultural-historical model of early childhood science education. En M. Fleer, N. Pramling (Eds.), A cultural-historical study of children learning science (pp. 199-213). Dordrecht: Springer.

Fragkiadaki G., Ravanis K. (2014) Mapping the interactions between young children while approaching the natural phenomenon of clouds creation. Educational Journal of the University of Patras UNESCO Chair 1(2), 112-122.

French L. (2004) Science as the center of a coherent, integrated early childhood curriculum. Early Childhood Research Quarterly 19(1), 138-149.

Fullan M. (1994) La gestión basada en el centro: el olvido de lo fundamental. Revista de Educación 304, 147-163. 
Fullan M. (2008) The six secrets of change. San Francisco: Jossey-Bass.

García S. (2008) La formación del profesorado de Educación Infantil. En M. R. Jiménez Liso (Ed.), XXIII Encuentros de la Didáctica de las Ciencias Experimentales (pp. 248-255). Almería: Universidad de Almería.

Glauert E. B. (2009) How young children understand electric circuits: Prediction, explanation and exploration. International Journal of Science Education 31(8), 1025-1047.

Hadzigeorgiou Y. (2002) A study of the development of the concept of mechanical stability in preschool children. Research in Science Education 32(3), 373-391.

Hecker J. (2008) La casa de los pequeños exploradores. Ariel: Barcelona.

Hickling A., Wellman H. (2001) The Emergence of Children's Causal Explanations and Theories: Evidence from Everyday Conversation. Developmental Psychology 37(5), 668683.

Kada V., Ravanis K. (2016) Creating a simple electric circuit with children between the ages of five and six. South African Journal of Education 36(2), 1-9.

Kamii C., Devries R. (1983) El conocimiento físico en la educación infantil. México DF: Ed. Siglo XXI.

Kaufman M., Fumagalli L. (2000) Enseñanza de Ciencias Naturales. Reflexiones y propuesta didácticas. Barcelona: Paidós.

Laguía M., Vidal C. (2008) Rincones de actividad en la Escuela Infantil (0 a 6 años). Barcelona: Graó.

Moreno M. (1986) Ciencia y Construcción del Pensamiento. Enseñanza de las Ciencias 4(1), 5763.

Museo Nacional de CyT (2007) Bienvenido al Pequemuseo. Madrid.

Museu de la Ciencia de la Caixa (2002) Experimentos con la electricidad. Aula, 110, 83-95.

Piaget J., Inhelder, B. (2000) The psychology of childhood. New York, NY: Basic Books.

Porlán R., Martín R. (2000) El diario como instrumento para detectar problemas y hacer explícitas las concepciones. En R. Porlán y J. Martín: El diario del profesor. Un recurso para la investigación en el aula (pp.21-69). Sevilla: Díada Editora. Recuperado de http://www.zona-bajio.com/diario_del_profesor.pdf

Pro A. (2008) Jugando con los circuitos y la corriente eléctrica. En la obra: El desarrollo del pensamiento científico y técnico en la Educación Primaria (pp. 43-82). Madrid: ISFP.

Pro A. (2011) El Practicum en el aula de ciencias (física y química): orientaciones para el diseño, experimentación y evaluación de actividades. En A. Caamaño (Coord.), Física y Química. Investigación, innovación y buenas prácticas (pp. 205-225). Barcelona: Graó.

Pro A., Rodríguez J. (2010) Aprender competencias en una propuesta para la enseñanza de los circuitos eléctricos en Educación Primaria. Enseñanza de las Ciencias 28(3), 385-406.

Pro A., Rodríguez J. (2011) La investigación en la Didáctica de las Ciencias Experimentales. Educatio Siglo XXI 29(1), 129-148.

Shepardson D. P., Moje D. B. (1994) The nature of fourth graders' understandings of electric circuits. Science Education 78, 489-514. 
Solomonidou C., Kakana D. M. (2000) Preschool Children's Conceptions about the Electric Current and the Functioning of Electric Appliances. European Early Childhood Education Research Journal 8(1), 95-111.

Sugrañes E., Alós M., Andrés N., Casal S., Castrillo C., Medina N., Yuste M. (2012) Observar para interpretar. Actividades de vida cotidiana para la educación infantil (2-6). Barcelona: Graó.

Tonucci F. (1997) La verdadera reforma empieza a los tres años. Investigación en la escuela 33, 516.

Vega S. (2006) Ciencias 0-3. Laboratorios de Ciencias en la escuela infantil. Barcelona: Graó.

Vega S. (2007) Ciencias 3-6. Laboratorios de Ciencias en la escuela infantil. Barcelona: Graó.

Vizcaíno I. M. (2008) Guía fácil para programar en Educación Infantil (0-6 años). Trabajar por proyectos. Madrid: Wolters Kluwer. 